\title{
RESEARCH HIGHLIGHT Novel SARS-CoV-2 receptors: ASGR1 and KREMEN1
}

Markus Hoffmann $\mathbb{D}^{1,2^{凶}}$ and Stefan Pöhlmann $\mathbb{D}^{1,2^{凶}}$

(c) CEMCS, CAS 2021

Cell Research (2022) 32:1-2; https://doi.org/10.1038/s41422-021-00603-9

The interaction of the SARS-CoV-2 spike protein with the cellular receptor ACE2 is believed to be essential for viral entry into host cells. In a recent paper published in Cell Research, Gu and colleagues report that ASGR1 and KREMEN1 can also serve as SARS-CoV-2 receptors and might impact viral target cell range and antibody-mediated neutralization.

The coronavirus spike (S) protein facilitates viral entry into cells. For this, the $S$ protein engages cellular receptors and the expression pattern and sequence of these receptors mainly determine which cells and species can be infected. A receptor binding domain (RBD), located in the surface unit, $S 1$, of the $S$ protein, interacts with receptors. The RBDs in the $S$ proteins of SARS-CoV and SARS-CoV-2, the causative agents of SARS and COVID-19, respectively, exhibit sequence homology and bind to angiotensin-converting enzyme 2 (ACE2) for host cell entry. ${ }^{1,2}$

In order to cause disease in mice, adaptation of SARS-CoV and SARS-CoV-2 to murine ACE2 or directed expression of human ACE2 in murine tissues is required and knockout of ACE2 strongly reduces viral spread. ${ }^{3}$ Further, susceptibility of cell lines to SARSCoV and SARS-CoV-2 infections largely correlates with ACE2 expression. However, this correlation is not absolute and a recent study reported ACE2-independent SARS-CoV-2 entry into a lung cell line. ${ }^{4}$ Moreover, SARS-CoV- $2,{ }^{5}$ like SARS-CoV, ${ }^{6}$ can infect a large spectrum of cells, tissues and organs in human patients and evidence for SARS-CoV-2 infection of cells expressing low levels of or no ACE2 has been documented. However, cellular factors that support ACE2-independent entry have largely remained elusive.

In a recent Cell Research paper, Gu and colleagues identified the cellular proteins asialoglycoprotein receptor-1 (ASGR1) and Kringle Containing Transmembrane Protein 1 (KREMEN1) as $\mathrm{S}$ protein-binding partners. ${ }^{7}$ Directed expression of ASGR1 and KREMEN1 in an ACE2 ${ }^{-}$cell line allowed for SARS-CoV-2 but not SARS-CoV entry (Fig. 1a). Similar results were obtained for SARSCoV-2 infection in a mouse model, although entry via ASGR1 and KREMEM1 was generally less efficient as compared to ACE2dependent entry.

Antibody inhibition and knockdown studies revealed that endogenous KREMEN1 or ASGR1 was, jointly with ACE2, required for SARS-CoV-2 entry into certain cell lines. Entry into a lungderived cell line, HTB-182, and a liver-derived cell line, Li7, was ACE2-independent and KREMEN1- (HTB-182 cells) or ASGR1dependent (Li7 cells), and was associated with resistance to neutralizing antibodies targeting the $S$ protein/ACE2 interface. Thus, ASGR1 and KREMEN1 are bona fide SARS-CoV-2 receptors that might protect the virus from certain neutralizing antibodies.
Analysis of clinical samples revealed that relative expression of ACE2, ASGR1 and KREMEN1 in the respiratory epithelium was higher in SARS-CoV-2-infected cells compared to uninfected cells. Further, expression of KREMEM1 in secretory cells correlated more strongly with susceptibility to infection than ACE2 expression. ASGR1- and KREMEN1-specific antibodies blocked $S$ protein binding and entry into cell lines and reduced infection of lung organoids, suggesting a role for these factors in SARS-CoV-2 infection of the respiratory tract.

Several cellular factors other than ACE2 have been reported to promote SARS-CoV and/or SARS-CoV-2 entry, including heparan sulfate proteoglycans ${ }^{8}$ and sialic acid-containing glycolipids. ${ }^{9}$ However, ASGR1 and KREMEN1 stand out because they fulfill the central criterion for a receptor-their expression renders cells susceptible to infection that are otherwise non-susceptible. This raises several interesting questions:

What are the "mechanics" of ASGR1- and KREMEN1-mediated entry? It has been proposed that ACE2 binding induces subtle conformational changes in the $\mathrm{S}$ protein that promote proteolytic activation. It seems unlikely that ASGR1 and KREMEN1 induce similar changes and $\mathrm{S}$ protein activation during ASGR1/KREMEN1dependent entry remains to be examined. A focus of these studies should be on TMPRSS2 vs cathepsin L dependence and the role of the restriction factors IFITM2/3.

How does SARS-CoV-2 S (SARS-2-S) engage ASGR1 and KREMEN1 and why does SARS-CoV S (SARS-S) fail to do so? Gu and coworkers show that not only the RBD but also the N-terminal domain (NTD) of SARS-2-S bind to ASGR1 and KREMEN1 (Fig. 1b). Therefore, the pronounced differences between the SARS-S and SARS-2-S NTD sequences might, at least in part, account for the failure of SARS-CoV to engage ASGR1 and KREMEN1 for entry.

Why is ASGR1- or KREMEN1-dependent entry associated with resistance against neutralization by antibodies? Again, binding of the SARS-2-S NTD to ASGR1 and KREMEN1 might hold the answer since these interactions should not be inhibited by the RBD-specific antibody 404-one of the two antibodies analyzed by Gu et al. The second antibody, 515, does not bind to the RBD but might target an S protein surface that can modulate ACE2 but not ASGR1/KREMEN1 binding. Experiments with additional antibodies and soluble ACE2 should provide clarity. Puray-Chavez et al. and Ramirez and colleagues reported that ACE2independent entry was not associated with neutralization resistance ${ }^{4,10}$ and the reasons for these discrepant results remain to be determined.

Does ASGR1 and KREMEN1 usage differ between SARS-CoV-2 variants? The virus used by Gu and coworkers has been circulating

\footnotetext{
${ }^{1}$ Infection Biology Unit, German Primate Center, Göttingen, Germany. ${ }^{2}$ Faculty of Biology and Psychology, Georg-August-University Göttingen, Göttingen, Germany.

凶email: mhoffmann@dpz.eu; spoehlmann@dpz.eu
} 


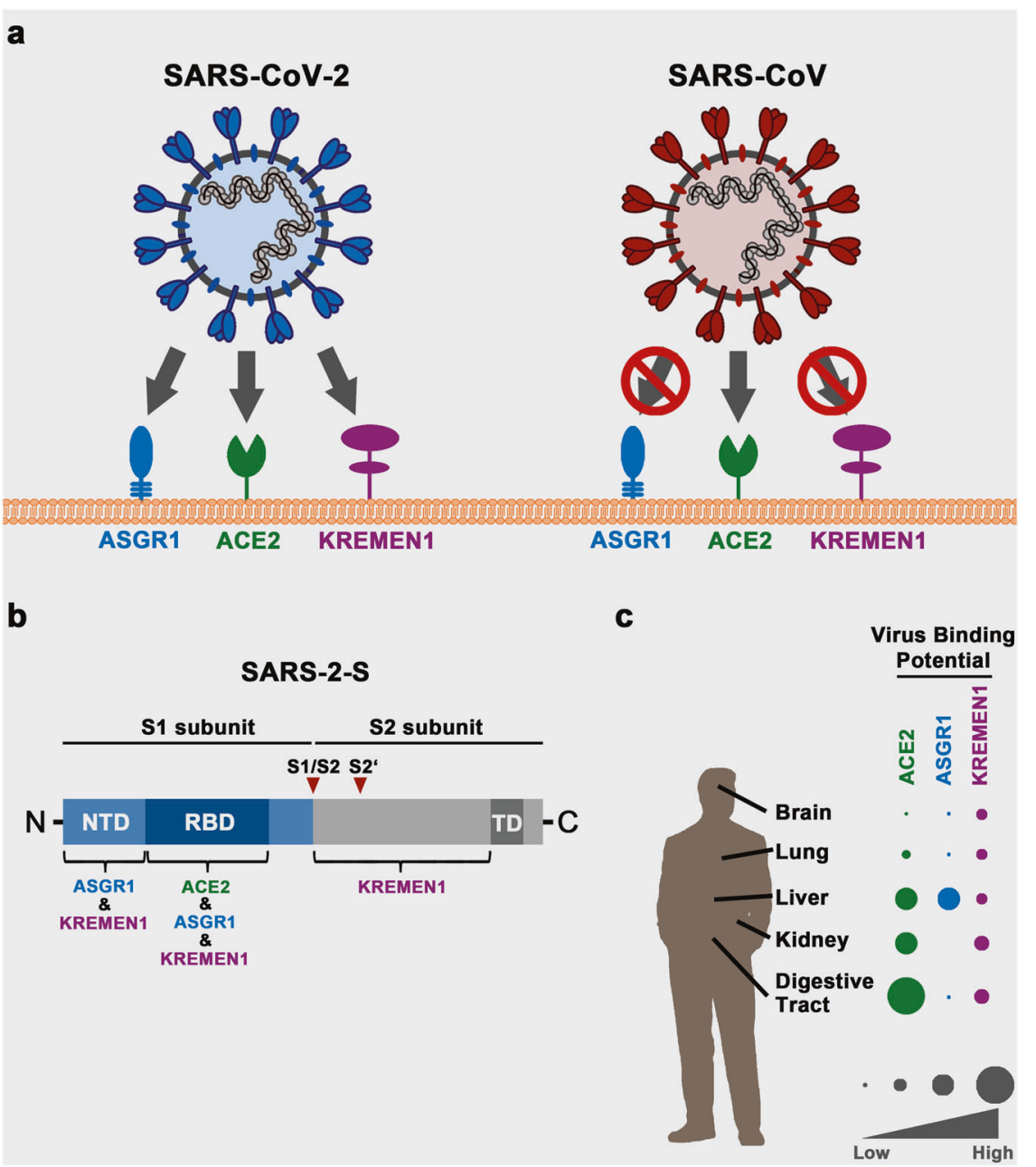

Fig. 1 Role of ASGR1 and KREMEN1 in SARS-CoV-2 infection. a SARS-CoV-2 can engage ACE2, ASGR1 and KREMEN1 as receptors for cell entry while SARS-CoV can only use ACE2. b The surface unit, S1, of the SARS-CoV-2 spike protein (SARS-2-S) contains an N-terminal domain (NTD) and a receptor binding domain (RBD). The RBD interacts with ACE2, ASGR1 and KREMEN1. The NTD can also bind to ASGR1 and KREMEN1, and the transmembrane unit, S2, can bind to KREMEN1. c Virus binding potential of tissues determined in silico based on receptor expression levels.

early in the pandemic. Therefore, it should be investigated whether viral variants that emerged later and harbor mutations in the S protein use ASGR1 and/or KREMEN1 more efficiently and whether this contributes to viral spread. Notably, ACE2independent entry has so far only been observed with mutated $S$ proteins, with mutation E484D playing an important role. ${ }^{4,10}$

How prominent is the contribution of ASGR1 and KREMEN1 to SARS-CoV-2 spread in humans? In silico analyses predicted that ACE2, ASGR1 and KREMEN1 may contribute to viral attachment to various tissues (Fig. 1C), and RNA-seq analyses of upper respiratory tract samples from COVID-19 patients provided evidence for infection of $\mathrm{ACE}^{-} / \mathrm{KREMEN1}{ }^{+}$epithelial and $\mathrm{ACE} 2^{-} / \mathrm{ASGR} 1^{+}$ immune cells. However, it is not clear whether particle uptake without productive infection was responsible for some of the signals. Although ASGR1 and KREMEN1 contributed to infection of lung organoids, ACE2 was more important. Further experimentation is thus required to clarify this question.

In sum, the identification of ASGR1 and KREMEN1 as SARS-CoV2 receptors advances our understanding of SARS-CoV-2 cell tropism and pathogenesis. Further, this finding demonstrates a previously unappreciated impact of the target cell type on antibody-mediated neutralization.

\section{REFERENCES}

1. Hoffmann, M. et al. Cell 181, 271-280 e278 (2020).

2. Zhou, P. et al. Nature 579, 270-273 (2020).

3. Bao, L. et al. Nature 583, 830-833 (2020).

4. Puray-Chavez, M. et al. Cell Rep. 36, 109364 (2021).

5. Puelles, V. G. et al. N. Engl. J. Med. 383, 590-592 (2020)

6. Gu, J. et al. J. Exp. Med. 202, 415-424 (2005).

7. Gu, Y. et al. Cell Res. https://doi.org/10.1038/s41422-021-00595-6 (2021).

8. Clausen, T. M. et al. Cell 183, 1043-1057 e1015 (2020).

9. Nguyen, L. et al. Nat. Chem. Biol. https://doi.org/10.1038/s41589-021-00924-1 (2021).

10. Ramirez, S. et al. Antimicrob. Agents Chemother. 65, e0009721 (2021).

\section{COMPETING INTERESTS}

The authors declare no competing interests.

\section{ADDITIONAL INFORMATION}

Correspondence and requests for materials should be addressed to Markus Hoffmann or Stefan Pöhlmann.

Reprints and permission information is available at http://www.nature.com/ reprints 